\title{
One Score to Rule Them All? ICU Scoring at the Dawn of the Digital Age*
}

\author{
Tobias M. Merz, MD \\ Department of Intensive Care Medicine \\ Inselspital, Bern University Hospital \\ University of Bern \\ Bern, Switzerland
}

S everity of illness scores were introduced in critical care during the 1980s and were quickly incorporated into medical practice (1-3). These scores contain data regarding health status and diagnosis, as well as physiologic and laboratory data, collected during the first 24 hours of ICU admission and aim to estimate patients' outcomes. In this issue of Critical Care Medicine, Deliberato et al (4) present results which indicate that small but significant deviations in WBC count, creatinine, and urea nitrogen exist, in the premorbid state, before ICU admission in obese compared with normal weight patients. The authors hypothesize that the degree of deviation of scoring variables from baseline to development of critical illness differs between obese and nonobese patients, even with similar severity of illness scores, and this potentially leads to severity of illness score misclassification.

Are these findings relevant to the critical care practitioner? At first glance, the magnitude in differences between premorbid baseline values may not seem important. However, ICU severity scores are used as the basis for various processes in the critical care context. They are used to characterize patients in terms of severity of illness in clinical studies, for the evaluation of ICU performance, in quality improvement initiatives and for benchmarking purposes. Even though ICU severity scores are not the key elements for deciding upon treatment for each individual patient, they have a role in clinical decision-making when triaging and in identifying patients with the potential for unexpected outcomes. As the prevalence of overweight patients and obesity generally is increasing globally (5), baseline imbalances could lead to severity of illness score misclassification and less accurate scoring for an important group of patients. ICU scores are based on model development decisions and processes, such as variable selection, that must occur in a programmable and automated manner. Therefore, the necessary categorization or dichotomization of

\footnotetext{
*See also p. 394.

Key Words: health information management; intensive care unit; severity of illness; severity of illness scores

Dr. Merz disclosed that his research group has received a grant from the Swiss National Science Foundation for a research project in the context of ICU big data analysis for the coming two years.

Copyright (C) 2018 by the Society of Critical Care Medicine and Wolters Kluwer Health, Inc. All Rights Reserved.
}

DOI: 10.1097/CCM.0000000000002900 some variables can affect estimation, depending on apparently arbitrary cut-off values. When applying scores, even small changes in continuous values can generate the threshold difference in scoring and lead to a shift in the classification of a patient, such as for a disease severity from mild to moderate or moderate to severe. In this context, and as data analysis assumes a more prominent role in the care of individual patients, the accumulation of many small effects stemming from differences across different subsets of ICU patients may affect the performance of models for prognostication or of decision support systems.

A growing number of ICUs use electronic patient data management systems to register, report, and manage all relevant patient-specific data. This includes not only patient characteristics, but also continuous measurements of organ function parameters, results of laboratory analyses, and parameters determined by therapeutic interventions. Electronic systems not only provide many advantages when monitoring critically ill patients, but also for the development or application of prognostic models. All the patient data are recorded accurately and with a much higher temporal resolution and stored in a single database. The data can be presented in a freely selectable manner, which enables problem-orientated presentation of changes of data over time, allowing for a more comprehensive, pattern-oriented data analysis. The collection of detailed information of many thousand ICU admissions results in massive quantities of data-often referred to as "big data" (6). By discovering associations and understanding patterns and trends within the data-as done by the authors using the Medical Information Mart for Intensive Care II database (7) — big data analytics have the potential to improve care, save lives, and lower costs (8). The work presented by Deliberato et al (4) demonstrates the use of large databases to refine existing severity of illness indices.

However, to capitalize on the full potential offered by indepth analysis of large ICU datasets using big data analytics or machine learning-based approaches, we must move away from the concepts used in conventional ICU scores. These scoring systems have served the ICU community well for both quality control and management of the ICU and can help identify patients with a generally higher risk of deterioration and the likelihood of serious adverse events during their ICU stay. Conventional ICU scores are nevertheless of little assistance for continuous structured assessment and management of individual patients due to the following characteristics:

1) The performance of conventional scoring systems might be suboptimal due to the nonsystematic selection of few predictor variables and the use of simplified correlations between predictors and outcome. 
2) The predicted outcome of established ICU scores is mortality and not occurrence of organ dysfunction. After the initial decision to admit the patient to the ICU for full treatment, the prediction of mortality by scoring is not of great importance for further treatment decisions.

3) For simplicity and feasibility of routine use, only the most pathologic value in a dataset obtained during first day of ICU admission is used for scoring, whereas changes of organ function variables over time-which may be of greater prognostic relevance-are not integrated.

4) Organ function parameters can be influenced by the established organ support or replacement modalities and changes in treatment intensity, which are not adequately included as scoring variables.

Despite the obvious potential and the promise of supporting a wide range of medical functions, including automated patient surveillance and clinical decision support, prognostic modeling based on "big data" using modern machine learning methods is not well established in an ICU context (9). To date, only basic predictive models for the detection of deterioration of organ functions have been developed for specific disease conditions, such as hemodynamic instability $(10,11)$, sepsis $(12)$, and lung failure (13). Given the complexity of the task, systems which include and integrate a multitude of patient data accumulating over time during the treatment process to predict the course of illness severity in the immediate future do not exist yet. However, the advent of large-scale healthcare information systems offers the opportunity to advance medicine to the digital age. Using this information smartly requires further extensive research and the collaboration of clinicians and data specialists as was exemplary done by Deliberato et al (4). To bring the potential of big data to the bedside, we will need to make patient data accessible for research, define clinically meaningful endpoints, and develop the right analytic methods.

\section{REFERENCES}

1. Capuzzo M, Valpondi V, Sgarbi A, et al: Validation of severity scoring systems SAPS II and APACHE II in a single-center population. Intensive Care Med 2000; 26:1779-1785

2. Higgins TL, Teres D, Copes WS, et al: Assessing contemporary intensive care unit outcome: An updated Mortality Probability Admission Model (MPMO-III). Crit Care Med 2007; 35:827-835

3. Moreno RP, Metnitz PG, Almeida E, et al; SAPS 3 Investigators: SAPS 3-From evaluation of the patient to evaluation of the intensive care unit. Part 2: Development of a prognostic model for hospital mortality at ICU admission. Intensive Care Med 2005; 31:1345-1355

4. Deliberato RO, Ko S, Komorowski M, et al: Severity of Illness Scores May Misclassify Critically III Obese Patients. Crit Care Med 2018; 46:394-400

5. Ng M, Fleming T, Robinson M, et al: Global, regional, and national prevalence of overweight and obesity in children and adults during 1980-2013: A systematic analysis for the Global Burden of Disease Study 2013. Lancet 2014; 384:766-781

6. Saeed M, Villarroel M, Reisner AT, et al: Multiparameter intelligent monitoring in intensive care II: A public-access intensive care unit database. Crit Care Med 2011; 39:952-960

7. Saeed M, Lieu C, Raber G, et al: MIMIC II: A massive temporal ICU patient database to support research in intelligent patient monitoring. Comput Cardiol 2002; 29:641-644

8. Moss TJ, Lake DE, Calland JF, et al: Signatures of Subacute Potentially Catastrophic Illness in the ICU: Model Development and Validation. Crit Care Med 2016; 44:1639-1648

9. Pirracchio R, Petersen ML, Carone M, et al: Mortality prediction in intensive care units with the Super ICU Learner Algorithm (SICULA): A population-based study. Lancet Respir Med 2015; 3:42-52

10. Cao H, Eshelman L, Chbat N, et al: Predicting ICU hemodynamic instability using continuous multiparameter trends. Conf Proc IEEE Eng Med Biol Soc 2008; 2008:3803-3806

11. Eshelman LJ, Lee KP, Frassica JJ, et al: Development and evaluation of predictive alerts for hemodynamic instability in ICU patients. AMIA Annu Symp Proc 2008; 2008:379-383

12. Lindner HA, Balaban Ü, Sturm T, et al: An algorithm for systemic inflammatory response syndrome criteria-based prediction of sepsis in a polytrauma cohort. Crit Care Med 2016; 44:2199-2207

13. Huddar V, Rajan V, Bhattacharya S, et al: Predicting postoperative acute respiratory failure in critical care using nursing notes and physiological signals. Conf Proc IEEE Eng Med Biol Soc 2014; 2014:2702-2705 